\title{
Desigualdades socioeconómicas en salud en la Argentina e Italia en 2015-2018
}

\section{Socioeconomic inequalities in health in Argentina and Italy in 2015-2018}

\section{Solange Rodríguez Espínola}

solange.rodriguezespinola@gmail.com

Pontificia Universidad Católica Argentina Santa

María de los Buenos Aires (UCA). Observatorio de la

Deuda Social Argentina (ODSA), Argentina

\section{Pilar Filgueira}

pfilgueirag@gmail.com

Pontificia Universidad Católica Argentina Santa María de los Buenos Aires (UCA). Observatorio de la Deuda Social Argentina (ODSA) / CONICET, Argentina

\section{Simone Sarti}

simone.sarti@unimi.it

Universidad de Milán, Italia (UNIMI). Departamento de Ciencias Sociales y Políticas, Italia

\section{Recepción: 19 Mayo 2020 \\ Aprobación: 27 Octubre 2020 \\ Publicación: 01 Febrero 2021}

Cita sugerida: Rodríguez Espínola, S., Filgueira, P. y Sarti, S. (2021). Desigualdades socioeconómicas en salud en la Argentina e Italia en 2015-2018. Cuestiones de Sociología, 24, e112. https://doi.org/10.24215/23468904e112
Resumen: En la literatura hay una falta de investigación acerca de las desigualdades en salud en Sudamérica y sus diferencias con respecto a las de los países desarrollados. Como en Italia se han registrado tendencias similares en los últimos años y muestra similaridades con la Argentina, se ha decidido utilizar el país mediterráneo con fines comparativos. La hipótesis planteada consistió en que, más allá de las diferencias estructurales, las desigualdades en salud presentarían patrones similares en ambos países, caracterizados por una economía capitalista. Los grupos sociales en posiciones educativas y ocupacionales favorables exhibirían un mejor estado de salud que los grupos desfavorecidos. Se presentaron algunas estadísticas descriptivas sobre la situación general en los dos países, y luego se analizaron datos provenientes de dos encuestas que recolectan información individual sobre condiciones sociales y estados de salud (EDSA, de 2017 a 2018, y "EHIS - European Health Interview Survey", ISTAT, 2015). Los resultados muestran que la Argentina e Italia poseen diferentes niveles de bienestar, tasa de mortalidad y servicios de salud, pero que las disparidades relativas en salud parecen muy similares, confirmando la hipótesis de Marmot (2017) sobre la forma general de las desigualdades en salud. Trabajadores manuales y precarios, particularmente las personas desempleadas, sistemáticamente presentan un peor estado de salud percibido con respecto a las clases sociales más altas.

Palabras clave: Argentina, Italia, Desigualdades en salud, Salud percibida, Estudios comparativos.

Abstract: In the literature there is a lack of investigation on health inequalities in South America and their differences with respect to those in the developed countries. Since Italy has recorded similar economic trends in recent years and has similarities with Argentina, we decided to use the Mediterranean country for comparative purposes. Our hypothesis was that, beyond structural differences, health inequalities present similar patterns in these two countries characterized by a capitalist economy. Social groups in advantaged educational and occupational positions exhibit better health than disadvantaged groups. We present some descriptive statistics on the overall situation in the two countries, and we then analyse data stemming from two surveys that collected individual information on social conditions and health statuses (OASD from 2017 to 2018, and "EHIS - European Health Interview Survey", ISTAT 2015). The findings show 
that Argentina and Italy have different levels of wellbeing, mortality rates, and health services. But relative disparities in health seem very similar, confirming the hypothesis of Marmot (2017) about the general form of health inequalities. Manual and precarious workers (in particular unemployed persons) present systematically worse perceived health with respect to higher social classes.

Keywords: Argentina, Italy, Health inequalities, Perceived health, Comparative studies.

\section{El rol de las condiciones socioeconómicas en las desigualdades en salud}

La investigación sobre las fuentes de heterogeneidad en la salud de la población es un tema central en las ciencias sociales y políticas. De modos distintos, la salud es considerada como el resultado principal del impacto de las condiciones sociales en la vida de los individuos, ya que se encuentra incrustada en el cuerpo humano a través de numerosas desventajas socioeconómicas que son acumuladas por los individuos a lo largo de sus vidas (Marmot, 2017; Cullati, Rousseaux, Gabadinho, Courvoiser \& Burton-Jeangros, 2014; DiPrete \& Eirich, 2006; Spencer \& Logan, 2002; Willson, Shuey \& Elder, 2007).

Muchos autores explican la variabilidad en salud a través de la aplicación de teorías estructuralistas, las cuales sostienen que el encontrarse en una posición social vulnerable incrementa la posibilidad de un mal estado de salud debido a estrés, morbilidad y mortalidad (Bartley, 2003; Drever, Daran \& Whitehead, 2004; Marmot, 2013; Phelan, Link \& Tehranifar, 2010; OMS, 2013; Wilkinson \& Marmot, 2003). En particular, estas teorías se enfocan principalmente en los factores socioeconómicos asociados a la posición social, a saber: niveles educativos, organización del mercado laboral, y recursos materiales como el ingreso, el ambiente laboral y las condiciones generales de trabajo.

Esta perspectiva asume que los individuos que ocupan diversas posiciones sociales están expuestos de modo distinto tanto al deterioro físico -agentes tóxicos, condiciones precarias de vivienda o trabajos peligrosos-como al desgaste psicológico -estrés debido a preocupaciones económicas, desempleo, carga laboral excesiva, tareas repetitivas y falta de autonomía laboral\#. Estos factores son considerados como determinantes de un amplio grupo de enfermedades (Cassel, 1976; Cohen, Janicki-Deverts \& Miller, 2007; Navarro, 1986; Phelan, Link \& Tehranifar, 2010; Siegrist \& Marmor, 2004).

La influencia que ejerce la posición socioeconómica del individuo sobre su salud frecuentemente no es directa, sino que es el producto de factores intermediarios: condiciones materiales - tales como la calidad de la vivienda-, circunstancias psicosociales -incluido el estrés- y comportamentales -como son el tabaquismo o la mala alimentación-. Este modelo incorpora al sistema de salud como otro determinante social ya que, por una parte, el deterioro o la mejoría del estado de salud tiene un efecto de retroalimentación sobre el estado socioeconómico, y por la otra, el sector de la salud juega un papel fundamental en la promoción y coordinación de las políticas de acción sobre los determinantes sociales. 
Así, de acuerdo con un extenso corpus de literatura científica, la posición social es un factor decisivo en la determinación de las condiciones de salud. La noción puede ser operacionalizada de diversas maneras; sin embargo, las dimensiones utilizadas con mayor frecuencia son el ingreso, el estatus ocupacional y el nivel educativo. Es preciso notar, además, que todas estas dimensiones se encuentran estrechamente correlacionadas. A continuación, se presentarán, brevemente, las características de cada una.

Un gran número de autores tienden a investigar la desigualdad utilizando el ingreso -frecuentemente expresado en quintiles o deciles- como el único indicador de la posición social de los individuos. Sin embargo, el ingreso no es capaz de revelar las dinámicas que producen y reproducen las desigualdades que conforman la estratificación social, en términos de ventajas materiales y simbólicas (Goldthorpe, 2010). En particular, la utilización del ingreso -o las escalas unidimensionales de estatus- resaltaría el gradiente entre la peor posición socioeconómica y de salud, pero impediría el reconocimiento de los distintos niveles de desigualdad atribuible al grupo ocupacional y al nivel educativo alcanzado, factores que se encuentran relacionados con la jerarquía de poder y con los recursos culturales.

Clasificar la posición ocupacional en el mercado laboral requiere la identificación de un grupo de individuos y familias que ocupen una posición similar dentro de las relaciones de poder que subyacen a la división social del trabajo y la posición en el mercado, así como dentro de las desigualdades relacionales y distributivas relacionadas. Operacionalmente, los individuos son clasificados de acuerdo con la típica situación laboral, que los diferencia en la relación de propiedad y autoridad en las que se encuentran inmersos (Erikson \& Goldthorpe, 1992). Por consiguiente, podrían estudiarse algunas hipótesis acerca de los modos en que las relaciones de poder que experimentan disparidades en el mercado laboral afectarían a la salud. Estas explicaciones no son mutualmente excluyentes, una no niega ni invalida a la otra, pero sí acentúan diferentes maneras en las que pertenecer a una clase determinada, y no a otra, podría resultar en un deterioro de la salud de sus miembros. $\mathrm{Al}$ re-adoptar la distinción propuesta por Bartley (2004), podrían considerarse, brevemente, tres modelos explicativos principales: (a) la explicación psicosocial, (b) la explicación materialista y (c) la acumulación de desventajas.

El principio básico de la explicación psicosocial, que es frecuentemente la más esgrimida por los distintos autores, es que las posiciones sociales sufren un estado de estrés sistemático, tal como lo define Aneshensel (1992), que produce un debilitamiento de las defensas inmunológicas y una menor protección frente a comportamientos riesgosos (Cohen, Janicki-Deverts \& Miller, 2007). Uno de los primeros autores en abogar por esta aproximación fue Karasek (1979), quien definió el estrés laboral como la tensión existente entre las demandas laborales y la autonomía en la toma de decisiones -laxitud/control de decisión del trabajo-. El estrés elevado resulta de una cuantiosa carga laboral asociada a una autonomía pobre en la toma de decisiones. Según Wilkinson (2002), la jerarquía de poder y la existencia de sistemas de desigualdad incrementan la carga de estrés en detrimento de las posiciones sociales más bajas. Por tanto, la pertenencia a una clase desfavorecida debería ser considerada como un factor de riesgo: es necesario teorizar el estatus social como un factor de riesgo psicosocial, 
y la biología dictamina que debe teorizárselo como una fuente de estrés crónico (Wilkinson, 2002).

En lo que respecta a la explicación psicosocial, debería mencionarse particularmente la aproximación de Siegrist $(1999 ; 2000)$, la cual se enfoca en el desbalance entre los esfuerzos y las recompensas. Esta aproximación fue inspirada por la teoría del intercambio de Homan, para la cual el modo en que cada persona se comporta se encuentra influido por experiencias pasadas, es decir, por cómo fue recompensada con anterioridad. La usure du travail resultaría en un déficit del balance de reciprocidad. En otras palabras, las personas frustradas con los roles sociales en términos de la habilidad de obtener recursos materiales, simbólicas y relacionales -ingresos, roles familiares, autoestima, etcétera- sufren de un déficit de recompensa social. La desfiguración causaría tal grado de estrés y sufrimiento que comprometería la eficiencia del sistema neurobiológico, por la exposición a un estado de estrés elevado y persistente.

La explicación materialista adjudica relevancia a las condiciones de vida materiales de los miembros de las clases más bajas, quienes dependen de menores ingresos y recursos de salud, y, por lo tanto, tienen un acceso limitado a los recursos que podrían salvaguardar su salud -por ejemplo, podrían no tener suficiente dinero para obtener medicamentos o asistencia sanitaria, podrían habitar viviendas inadecuadas sin calefacción, agua potable o cloacas, o podrían carecer de la ingesta calórica diaria para una vida saludable-. El rol de la explicación materialista es particularmente evidente al cotejar las desigualdades en salud a nivel internacional, por ejemplo, al comparar tasas y causas de mortalidad infantil en naciones particularmente desfavorecidas -como países subsaharianos o subdesarrollados- con aquellas naciones industrialmente avanzadas (OMS, 2015). Otro aspecto al que la explicación materialista atribuye una gran importancia es la mayor exposición de los miembros de las clases desfavorecidas a entornos insalubres, tal como lo demuestran los trabajadores de ciertos sectores industriales como la construcción, en los que los empleados se encuentran más expuestos a accidentes laborales (Karjalainen \& Niederlaender, 2004).

La tercera explicación refiere al modelo del curso vital (Sarti \& Zella, 2016; Cullati, Rousseaux, Gabadinho, Courvoiser \& Burton-Jeangros, 2014; Kuh \& Ben-shomo, 1997) y toma en cuenta el hecho de que encontrarse en el escalafón más bajo de la jerarquía social produce desventajas iniciales que se van acumulando a lo largo de la vida -desventajas que ya podrían presentarse durante el embarazo- (Turkheimer, Haley, Waldron, D'Onofrio \& Gottesman, 2003; Tucker-Drob. Rhemtulla, Harden, Turkheimer \& Fask, 2011). Más que una alternativa a las dos hipótesis expuestas anteriormente, esta explicación intenta echar luz sobre el proceso que produce y reproduce desigualdades en salud. En esta línea, debería notarse que los efectos de la selección no pueden ser apriorísticos, por lo que los sujetos que tienen menos probabilidades de desarrollar peores condiciones de salud -ansiedad, falta de autoestima, enfermedades variadas, etcétera- son también aquellos que más fácilmente ascienden la escalera de liderazgo social para ocupar las primeras posiciones. Desde un punto de vista de evidencia empírica, sin embargo, debería notarse que tales efectos de selección son considerados modestos (Aittomäki, 
Martikainen, Laaksonen, Lahelma \& Rahkonen, 2012; Mulatu \& Schooler, 2002).

Otra dimensión fundamental de la estratificación socioeconómica es la educación, la cual es un predictor confiable de la salud y se encuentra estrechamente asociada con bienestar, estilo de vida insalubre, morbilidad y mortalidad (Dupre, 2008; Eikemo, Huisman, Bambra \& Kunst, 2008; Ross \& Mirowsky, 1999; Kitigawa \& Hauser, 1973; Ross \& Wu, 1996). La educación es un predictor tan certero que los modelos que la utilizan como variable de control perciben un debilitamiento de los efectos de la clase ocupacional. Las explicaciones sobre el rol de la educación en la salud se superponen parcialmente con las de la posición ocupacional -también se encuentran firmemente vinculadas-. Los individuos que poseen una educación superior tienden a obtener mejores empleos -más saludable, menos estresante, más autónomo- (Della Bella, Sarti, Lucchini \& Bordogna, 2011; Brunner \& Marmot, 2006; Wilkinson \& Marmot, 2003), tienen mejores ingresos, pueden proporcionarse mejores viviendas y estándares de vida -como actividad física, mejores dietas, etcétera- (De Irala-Estévez, Groth, Johansson, Oltersdorf, Prättälä \& Martínez- González, 2000; Mancino, Lin \& Bellenger, 2004; McLaren, 2007), pueden contar con relaciones sociales más valiosas -con médicos o personas informadas-. Además, poseer mayores recursos culturales proporciona un mejor conocimiento de medicina y una utilización más fácil y efectiva de los servicios de salud, incluyendo la medicina preventiva (Herzlich \& Adam, 1994). Desafortunadamente, el único modo en que pueden estudiarse dinámicamente los cursos vitales es mediante costosos datos longitudinales, es decir, encuestas de panel.

Con este marco teórico en mente, en los análisis que siguen se investigan las desigualdades sociales en salud en la Argentina e Italia. Primero, se considera la diferencia general en varios indicadores de salud agregados, en ambos países. A continuación, se detallan las desigualdades en salud utilizando los datos de encuestas que permiten tomar en cuenta los estatus socioeconómicos específicos basados en condiciones ocupacionales y educacionales, y los estados de salud asociados.

La Argentina e Italia son países modernos, con distintos niveles de industrialización, logros educativos, bienestar, mortalidad y atención sanitaria. Sin embargo, la organización general de ambas sociedades es bastante similar: son países democráticos con economías capitalistas. Además, comparten cierta proximidad cultural, ya que un gran número de argentinos son descendientes de inmigrantes italianos. Por último, y lo más importante, ambos países han experimentado una tendencia macroeconómica similar durante los últimos años, ya que han sido duramente impactados por la crisis global que comenzó en el 2007, con un empobrecimiento relativo de la riqueza per cápita.

Asimismo, es interesante comparar el patrón de desigualdades sociales en salud en las dos naciones, que poseen un mercado laboral similar, pero con niveles de riqueza disímiles. En general, podrían ser utilizados como un caso de estudio para comparar las diferencias estructurales entre las sociedades sudamericanas y europeas. 


\section{Una comparación general de la salud en la Argentina e Italia}

La crisis económica global que se expandió desde los Estados Unidos en 2007 ha tenido un mayor impacto en Italia que en la Argentina; en particular, la tasa de desempleo oficial en Italia aumentó del 6,1 en 2007 al 11,9 en 2015, mientras que en la Argentina se mantuvo estable alrededor del 7 \% (Banco Mundial, 2016). También debe tenerse en cuenta que, a principios de siglo XXI, la Argentina ya había sufrido una dramática recesión económica (Dalle, 2017; Sandleris \& Wright, 2014). En lo que respecta al contexto sudamericano, la mayoría de las sociedades de Latinoamérica fueron marcadas por el subdesarrollo y por desigualdades severas. A mediados del siglo XX, la sociedad argentina parecía esbozar una alternativa: alta urbanización, empleo pleno, atención de salud y educación universales, industrialización intermedia avanzada y una extensa clase media - una sociedad relativamente integrada con desigualdad moderada y mucha movilidad social-. Pero esta sociedad cambió dramáticamente y tuvo que abandonar su anhelado futuro de progreso. En efecto, particularmente a fines de siglo $\mathrm{XX}$, en el contexto de reformas estructurales neoliberales (Collins, McCartney \& Garnham, 2015), la sociedad argentina no pudo evitar la trampa del subdesarrollo: la liberalización económica, la apertura comercial y la flexibilización financiera resultaron en inestabilidad, aumento del desempleo, pobreza y marginalización social, junto con un deterioro de la salud y educación pública y de la protección social. Estos procesos tuvieron como resultado una sociedad marcada por profundas desigualdades, conflictos internos y malestar social, un ciclo que produjo la crisis económica, social y política de 2001-2002, la más grave en la historia argentina reciente. En contraste, la década que siguió a la crisis, auxiliada por un contexto internacional favorable, evidenció que alguna recuperación económica, ocupacional, social, política e institucional era posible. Pero este período no duró demasiado: la economía se estancó, y nuevamente se reveló la fragmentación estructural de la sociedad. En 2015, la sociedad argentina presentó varias capas diferentes de segmentos marginalizados, pobres y excluidos.

No obstante, en años recientes, Italia y la Argentina han manifestado una tendencia similar en su evolución económica: ambos países han sufrido una recesión económica, con un estancamiento de la riqueza per cápita en comparación con las economías líderes, como Estados Unidos o Alemania. En la Argentina, el PBI per cápita (PPA constante 2011 en dólares de EE.UU.) de 2007 a 2015 se mantuvo por debajo de los 20 mil dólares; en el mismo período, el PBI italiano disminuyó de 39 a 34 mil dólares (Banco Mundial, 2016).

Mientras que la evolución económica reciente es similar en la Argentina e Italia, los dos países presentan niveles de bienestar marcadamente disímiles. Los indicadores de salud claramente muestran diferentes estándares de expectativa de vida y asistencia sanitaria, que imitan los amplios contrastes entre Europa y Sudamérica.

Las estadísticas de la OMS ayudan a describir los estados generales de salud en ambos países. En Italia, la esperanza de vida al nacer era de 83 años en 2012, mientras que en la Argentina era de 76; la esperanza de vida a los 60 años era de 25 en Italia y de 21 en la Argentina. Además, la esperanza de vida con buena salud era de 73 años en Italia y de 67 en la Argentina. El incremento de la esperanza de vida al nacer durante el período 2000-2012 fue de 3 años para Italia y de 2 para 
la Argentina. En 2013, la tasa de mortalidad de menores de cinco años - por cada 1000 nacidos vivos- fue de 4 en Italia y 13 en la Argentina.

Las principales causas de mortalidad son las mismas en los dos países: enfermedades isquémicas del corazón e infarto. Pero en la Argentina son relativamente más frecuentes las enfermedades respiratorias agudas con respecto a Italia, donde las muertes son ocasionadas mayormente por distintos tipos de cáncer y enfermedades cardiovasculares, información que es susceptiblemente diferente a partir de la pandemia de COVID-19 ya que aún no se terminan de precisar las causas de muerte diferenciando entre Italia y Argentina. También debe notarse que las diferencias en las causas de mortalidad sugieren distintos impactos del sistema del cuidado de la salud. En esta línea, la evidencia empírica más reciente, reportada por The Lancet, sostiene que el desempeño en el índice HAQ y las causas individuales de muerte mostraron patrones distintivos por región y nivel de desarrollo; aun así, han surgido heterogeneidades sustanciales por varias causas -incluyendo cánceres en los países con SDI más alto, enfermedad crónica del riñón, diabetes, enfermedades diarreicas, e infecciones respiratorias bajas en países con SDI medio (Barber Fullman, N., Sorensen, R. J., Bollyky, T., McKee, M., Nolte, E. et al., 2017).

En 2012, el porcentaje de PBI dedicado a la asistencia en salud fue de 9,3\% en Italia, mientras que en la Argentina apenas fue de $5 \%$.

En lo que respecta a estilos de vida insalubres, según el Observatorio Mundial de la Salud la prevalencia de obesidad en 2016 -en la población adulta- fue más alta en la Argentina (28,5\%) que en Italia (22,9\%), mientras que el consumo diario de tabaco estimado en el año 2015-en mayores de 15 años- fue ligeramente mayor en Italia (aproximadamente $20 \%$ ) que en la Argentina (16,3 \%) (OMS, 2017).

En el contexto europeo, las desigualdades en salud que presenta Italia son similares a las de otros países mediterráneos, menores que las de los países escandinavos y muy inferiores a las de los países de Europa del Este. Algunas hipótesis explicativas incluyen estilos de vida saludables - dieta mediterránea-, la presencia de una provisión del bienestar familiar generalizada y un sistema de salud público universalista. Estos factores parecen proteger a los italianos que se encuentran en desventaja, o reducir las disparidades en salud, mejor que en otros países europeos. Sin embargo, las desigualdades en salud en Italia son substanciales.

Se ha observado la desigualdad en la esperanza de vida considerando el nivel educativo. Un hombre italiano sin ningún título tiene 5,2 años menos de esperanza de vida que uno con un nivel educativo superior. Entre las mujeres, la diferencia es de 2,7 años (Fuente: ISTAT, 2019).

En Argentina, la Encuesta Nacional de utilización y gasto en servicios de salud (2012) reporta que, en 2010, un $14 \%$ de la población declaró que su salud era regular, mala o muy mala; $38,5 \%$ dijo contar con una buena salud, mientras que el restante $47,5 \%$ manifestó que su salud era muy buena o excelente. Apenas menos de la mitad de las personas encuestadas, el 46,8 \%, declaró que un doctor le había diagnosticado una enfermedad crónica. La percepción de un estado de salud deficiente y el diagnóstico empeoraban en la medida en que la edad de los encuestados aumentaba. La percepción de un buen estado de salud se incrementaba a mayor nivel educativo, mientras que el diagnóstico disminuía. 
Un patrón similar se observa al analizarse las respuestas según nivel de ingreso familiar y clase social.

Dos tercios de los entrevistados contaban con un sistema de protección de la salud. En todos los servicios de salud, incluyendo el uso y compra de medicamentos, predominaban las mujeres y el grupo de mayor edad -60 años y más-. En general, la utilización de los servicios de salud era levemente menor en aquellos con menores niveles educativos, con la excepción de la consulta médica y el uso de medicinas, casos en los que tendía a predominar el quintil con menos ingresos.

La evaluación del estado de la salud, de acuerdo con el diagnóstico de enfermedades crónicas y la salud autopercibida, muestra que la probabilidad de presentar un estado de salud deficitario se encuentra más relacionada con las mujeres y las personas mayores. Por su parte, la probabilidad de sufrir de mala salud disminuye a medida que el nivel educativo y el estatus socioeconómico aumentan.

Los datos del Observatorio de la Deuda Social Argentina aportan al análisis de los sistemas y recursos de protección social que tienen un impacto en el modo desigual en que los individuos logran cubrir el cuidado de su salud y sus necesidades de cuidado (Rodríguez Espínola, 2017). Los resultados reportados sugieren que la heterogeneidad y desigualdad social que prevalecen en la Argentina se proyectan en las condiciones de salud de los individuos y en la fragmentación y diversidad del sistema que los atiende (Schiavone, Rodríguez Espínola, Seoane y Ríos, 2015).

\section{Hipótesis}

Al considerar la evidencia empírica, Marmot (2017) resaltó que los países de altos y medianos ingresos tienden a presentar caminos similares en la construcción de heterogeneidad en salud. El Banco Mundial ha clasificado a Italia como un país de altos ingresos y a la Argentina como un país de ingresos medio-altos. Italia es definida como un país post-industrial con un nivel relativamente alto de actividades terciarias avanzadas, mientras que la Argentina presenta una desindustrialización prematura (Rodrik, 2016). El nivel de riqueza de ambos países es considerablemente diferente, y es probable que las desigualdades en salud repliquen esto.

La hipótesis que sostiene este estudio es que, a pesar de las diferencias en lo que respecta a la riqueza absoluta, la organización jerárquica del mercado laboral y la estructura económica general son bastante similares en los dos países, de modo que el gradiente entre salud y condiciones socioeconómicas, en términos relativos, es análogo. Tal como se discutió en la primera sección, los autores citados sostienen que los determinantes de las desigualdades en salud subyacen principalmente en la estructura socioeconómica, viéndose afectado también el proceso de reproducción intermediaria de las disparidades de salud, estrés constante, privación material, acumulación de desventajas a lo largo del curso de la vida y déficit de capital cultural. Conjeturamos que los grupos sociales que cuentan con ventajas educativas y ocupacionales, asociadas con mayores recursos materiales, socioeducativos e inmateriales -mayor ingreso, mejor prestigio laboral, autonomía laboral, etc.- tienden a poseer un mejor 
estado de salud que aquellos grupos en peores condiciones -desempleo, trabajo vulnerable o marginal, etcétera-.

Así, se pretende comparar las desigualdades en la salud entre la Argentina e Italia - a partir de indicadores subjetivos de salud de la población y su relación con variables socioeconómicas.

\section{Datos y metodología: la construcción de un conjunto de datos e indicadores comparables}

Para describir las desigualdades en salud en la Argentina e Italia, es necesario identificar indicadores empíricos de las condiciones sociales y los estados de salud.

Como medida de la salud se utilizó la salud autopercibida declarada por los argentinos e italianos consultados en dos importantes encuestas nacionales. La literatura socio-epidemiológica sostiene que la autoevaluación de la salud es una medida confiable $-\mathrm{y}$ un buen indicador- de las condiciones de salud reales (Drever, Daran \& Whitehead, 2004; Idler \& Benyamini, 1997; Johnson \& Wolinsky, 1993; Jylhä, 2009). Actúa como un constructo latente que combina la experiencia personal de síntomas agudos y crónicos junto con diferentes estados psicofísicos, como fatiga y dolores de espalda y de cabeza. Los estudios empíricos enfatizan que la salud auto-reportada también se encuentra asociada con las observaciones clínicas -como, por ejemplo, examinaciones físicas y presencia de morbilidad-y predice la red de mortalidad de enfermedades agudas y crónicas, discapacidades físicas y comportamientos de salud. Es más, la salud autopercibida es sugerida con fines comparativos por la OMS y se encuentra presente, efectivamente, en todas las encuestas individuales que indagan sobre temas de salud.

Para una evaluación de la robustez del análisis, se utilizó otro índice de percepción basado en las condiciones psicológicas del encuestado durante las últimas cuatro semanas. Consistió en una puntuación estándar basada en dos factores latentes, estimados separadamente para la Argentina e Italia. Se aplicó un análisis factorial para extraer dos factores estandarizados desde una batería de siete ítems comparables (ver Tabla 1 para más detalles). Aunque los ítems no eran exactamente iguales, pero sí semánticamente muy similares, se estimó que el factor latente sería capaz de captar la información acerca de la condición psicológica de los encuestados. Se utilizó el índice como variable métrica, una medida representativa de la distribución relativa del malestar psicológico en las dos poblaciones examinadas. 
Tabla 1

Tabla 1

Armonización de indicadores

\begin{tabular}{|c|c|c|c|c|}
\hline \multirow{2}{*}{ Indicador } & \multicolumn{2}{|c|}{ Preguntas de los cuestionarios } & \multirow{2}{*}{ Armonización } & \multirow{2}{*}{$\begin{array}{l}\text { Variables en el conjunto de datos } \\
\text { comparable }\end{array}$} \\
\hline & Argentina & Italia & & \\
\hline $\begin{array}{l}\text { Estado de salud } \\
\text { percibido por el } \\
\text { respondente }\end{array}$ & $\begin{array}{l}\text { "En general, ¿cuál es su estado de } \\
\text { salud?" } 0 \text { "Sin problemas", } 1 \text { "Algunos } \\
\text { problemas", } 2 \text { "Problemas crốnicos o } \\
\text { serios" }\end{array}$ & $\begin{array}{l}\text { "¿Cómo es su salud, en general?" De } \\
1 \text { "Muy buena" a } 5 \text { "Muy mala" }\end{array}$ & $\begin{array}{l}\text { ITALIA: De } 1 \text { a } 3 \text { = "Buena". De } 4 \text { a } 5 \\
\text { = "No buena" } \\
\text { ARGENTINA: } 0=\text { "Buena". De } 1 \text { a } 2= \\
\text { "No buena" }\end{array}$ & $0=$ Buena, $1=$ No buena \\
\hline $\begin{array}{l}\text { Condiciones } \\
\text { psicológicas en } \\
\text { las últimas } 4 \\
\text { semanas }\end{array}$ & $\begin{array}{l}\text { Cansado sin motivo. Nervioso. } \\
\text { Desesperanzado. Inquieto o } \\
\text { impaciente. Deprimido. Todo me } \\
\text { cuesta mucho esfuerzo. Tan triste que } \\
\text { nada puede alegrarme (1 Siempre, } 2 \\
\text { Muchas veces, } 3 \text { A veces, } 4 \text { Pocas } \\
\text { veces, } 5 \text { Nunca) }\end{array}$ & $\begin{array}{l}\text { Poco interés o placer en hacer las } \\
\text { cosas. Sentirse triste, deprimido o } \\
\text { desesperado. Tener problemas para } \\
\text { conciliar el sueflo o dormir toda la } \\
\text { noche sin despertarse, o dormir } \\
\text { demasiado. Sentirse cansado o tener } \\
\text { poca energía. Muestra una baja } \\
\text { opinión de sí mismo, siente que es un } \\
\text { fracaso o que se ha decepcionado a } \\
\text { si mismo o a su familia. Dificultad para } \\
\text { concentrarse en algo, como leer el } \\
\text { periódico o mirar televisión. Moverse } \\
\text { o hablar tan lentamente que otras } \\
\text { personas puedan notarlo, o sentir } \\
\text { inquietud o agitación inusual (1 Todos } \\
\text { los dias, } 2 \text { Más de la mitad de los } \\
\text { dias, } 3 \text { Algunos dias, } 4 \text { Nunca) }\end{array}$ & $\begin{array}{l}\text { Dos factores latentes estandarizados } \\
\text { fueron calculados por separado para } \\
\text { Italia y Argentina, con un análisis } \\
\text { factorial basado en los siete } \\
\text { indicadores originales. Sólo un factor } \\
\text { fue mas alto que un autovalor en } \\
\text { ambos paises. Para Italia, el factor } \\
\text { explicó el } 49.5 \% \text { de la varianza, } \\
\text { mientras que para Argentina explicó } \\
\text { un } 55.6 \%\end{array}$ & $\begin{array}{l}\text { Un indice de malestar psicológico, } \\
\text { basado en una puntuación estándar, } \\
\text { fue calculado separadamente en } \\
\text { ambos paises. }\end{array}$ \\
\hline $\begin{array}{l}\text { Clase social, en } \\
\text { bease a las } \\
\text { condiciones } \\
\text { œcupacionales } \\
\text { en el hogar }\end{array}$ & $\begin{array}{l}\text { "Estatus socio-económico del hogar" } \\
1 \text { Clase media profesional, } 2 \text { Clase } \\
\text { media no profesional, } 3 \text { Clase } \\
\text { trabajadora integrada, } 4 \text { Clase } \\
\text { marginal }\end{array}$ & $\begin{array}{l}\text { "Posición ocupacional más alta de los } \\
\text { hogares" 0 Profesionales, } 1 \\
\text { Empresarios, } 2 \text { Administrativos, } 3 \\
\text { Propietarios de pequeñas empresas, } \\
4 \text { Clase trabajadora, } 5 \text { Retirados, } 6 \\
\text { Desempleados, } 9 \text { Otras condiciones }\end{array}$ & $\begin{array}{l}\text { ITALIA: } 0=\text { Profesionales, De } 1 \text { a } 2= \\
\text { Clase media, De } 3 \text { a } 4=\text { Clase } \\
\text { trabajadora, } 6=\text { Clase marginal, } 5 \text { y } 9 \\
\text { = Casos perdidos. } \\
\text { ARGENTINA: Igual a los originales }\end{array}$ & $\begin{array}{l}1 \text { Clase media-alta y profesionales, } 2 \\
\text { Clase media, } 3 \text { Clase trabajadora } \\
\text { (empleo estable), } 4 \text { Clase marginal } \\
\text { (desempleo y condiciones precarias } \\
\text { de empleo) }\end{array}$ \\
\hline $\begin{array}{l}\text { Educación del } \\
\text { respondente }\end{array}$ & $\begin{array}{l}\text { "Máximo nivel educativo alcanzado" } 0 \\
\text { Sin título (< } 7 \text { años de educación), } 1 \\
\text { Nivel primario ( } 7<12 \text { años de } \\
\text { educación), } 2 \text { Nivel secundario (12< } \\
17 \text { años de educación), } 3 \text { Nivel } \\
\text { universitario ( } 17+\text { años de educación) }\end{array}$ & $\begin{array}{l}\text { "Máximo nivel educativo alcanzado" } 0 \\
\text { Sin título (<5 años de educación), } 1 \\
\text { Nivel primario ( }<8 \text { años de } \\
\text { educación), } 2 \text { Nivel secundario (13 < } \\
18 \text { años de educación), } 3 \text { Nivel } \\
\text { universitario (18+ años de educación) }\end{array}$ & $\begin{array}{l}\text { Para ITAUA y ARGENTINA: }(0=0) \\
(1=1)(2=2)(3=3)\end{array}$ & $\begin{array}{l}0 \text { Sin titulo (0), } 1 \text { Nivel primario (I), } 2 \\
\text { Nivel secundario (II), } 3 \text { Nivel terciario } \\
\text { (III) }\end{array}$ \\
\hline
\end{tabular}

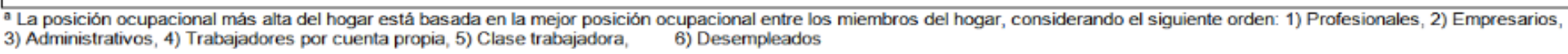

Todos los análisis de la población argentina utilizaron datos de la EDSA (Encuesta de la Deuda Social Argentina), que recaba datos anualmente en una muestra probabilística nacional urbana de adultos residentes en Argentina, de 18 años y más (Rodríguez Espínola, 2017). Teniendo en cuento los datos censales del año 2010, los participantes fueron seleccionados mediante un muestreo aleatorio polietápico por conglomerados, condiciones de vivienda y estatus socioeconómico. En la primera etapa, se utilizaron criterios demográficos para seleccionar las aglomeraciones urbanas - por región geográfica y tamaño-. Después, un muestreo aleatorio estratificado fue utilizado junto a un trazado de radio variable, con la probabilidad proporcional al tamaño de la población de 18 años y más. Un encuestador entrenado visitó los hogares, e individuos de cada uno de ellos, aleatoriamente seleccionados por cuotas de sexo y edad, fueron invitados a participar. Todas las respuestas fueron ponderadas para reflejar la verdadera proporción de cada sub-grupo en la totalidad de la población argentina. El protocolo utilizado fue aprobado por una comisión de revisión institucional en ética de la Pontificia Universidad Católica Argentina, y todos los sujetos participantes brindaron oralmente su consentimiento informado.

Con el fin de lograr un mayor número de observaciones en la Argentina, se utilizaron las encuestas de 2017 y 2018 (EDSA-Serie Agenda para Equidad), con un total de 11.417 casos.

En Italia, la encuesta similar a la EHIS es la European Health Interview Survey conducida por ISTAT (Instituto Nacional de Estadísticas), en acuerdo con EUROSTAT en 2015. El cuestionario de la encuesta recolectó información 
sobre características sociodemográficas, condición ocupacional, condiciones de salud y utilización de los servicios de salud. La muestra tuvo un diseño de estratificación doble, con un primer nivel constituido por las municipalidades; dentro de ellas, los hogares fueron seleccionados aleatoriamente. Todos los miembros del hogar fueron entrevistados.

Para el análisis de los datos de ambas encuestas, se seleccionó a los adultos de entre 30 y 60 años de edad. Se excluyó a los menores de 30 años porque podrían no haber terminado la escuela, lo que causaría un problema de heterogeneidad en la atribución del nivel educativo. Por otra parte, no se incluyó a los mayores de 60 años por problemas de selección (Beckett, 2000; Willson et al., 2007).

Comparar ambas encuestas implicó dos problemas principales: el diseño muestral y la operacionalización de los indicadores relevantes. El primer problema fue resuelto mediante una selección aleatoria de un miembro por hogar en la muestra italiana. Esta selección igualó estructuralmente a ambas muestras. Al final de este proceso de limpieza de datos, se conservaron 5.823 casos válidos para la Argentina y 5.655 para Italia.

Por otra parte, la solución del segundo problema fue más complicada, ya que las preguntas de los cuestionarios eran distintas en algunos casos. En particular, se encontraron diferencias problemáticas en las clasificaciones de condiciones socioeconómicas, calificaciones educativas y estado de salud. Luego de una reflexión teórica y de algunos controles empíricos, se logró una armonización satisfactoria de los indicadores. La clasificación resultante de las variables puede observarse en la última columna de la Tabla 1. La pregunta que sirvió de guía al presente trabajo fue la comparación de las desigualdades en salud en la Argentina e Italia. Por lo tanto, se buscó describir las asociaciones entre los grupos sociales definidos sobre la base de las condiciones ocupacionales y educacionales y del estado de salud.

La Tabla 2 muestra algunas diferencias en la estructura ocupacional de ambos países. El segmento de la población que se encuentra en condiciones marginales es el doble en la Argentina que en Italia ( $16 \%$ vs. $7 \%$ ), mientras que la clase media es más extensa en el país europeo (14 p.p. mayor). La clase media-alta y profesionales es más numerosa en la Argentina ( $14 \%$ vs. $6 \%$ ), si bien la condición marginal es representada por el $16 \%$ de la muestra argentina y el $7 \%$ de la italiana. 
Tabla 2

Tabla 2

Estadisticos descriptivos, porcentajes

\begin{tabular}{|l|cc|}
\hline \multicolumn{1}{|c|}{$\%$} & Argentina & Italia \\
\hline Salud percibida & 62,4 & \\
Buena & 37,6 & 65,6 \\
No buena & std & 34,4 \\
Índice de malestar psicológico & & std \\
(de 1-peor, a 7-mejor) & 53,9 & \\
Género & 46,1 & 49,9 \\
Femenino & & 50,1 \\
Masculino & 6,8 & \\
Nivel educativo & 32 & 0,5 \\
Sin título (0) & 29,7 & 34,7 \\
Nivel primario (1) & 31,5 & 48,2 \\
Nivel secundario (2) & 16,3 & 16,6 \\
Nivel terciario/universitario (3) & 37,9 & 7,1 \\
Clase social & 32,0 & 40,6 \\
Marginal & 13,8 & 46,4 \\
Clase trabajadora & 5823 & 6,0 \\
Clase media & & 5655 \\
Clase media-alta y profesionales & & \\
\hline Casos válidos & & \\
\hline
\end{tabular}

En lo que se refiere a la segmentación italiana, se observa una estructura similar al resto de los países europeos. En particular, existen unas clases media y trabajadora principal -que agregadas alcanzan al $87 \%$ de la población-, una clase alta más pequeña y una clase marginal que se incrementó en los últimos años de recesión económica, debido al crecimiento del desempleo (Sarti \& Zella, 2016). Sin embargo, el caso argentino es más interesante debido a su ausencia en los debates de la literatura internacional.

Los datos muestran que la estructura social actual de la Argentina es más heterogénea y desigual que en Italia. La clase media-alta incluye tanto a directores corporativos, profesionales, empresarios, productores agropecuarios y comerciantes medianos, como a técnicos calificados y empleados de los sectores más dinámicos de la economía. Estos sectores sociales se encuentran integrados a la cultura occidental, presentando una calidad de vida y patrones de consumo similares a los de las clases medias del sur de Europa. Muchos de estos individuos se concentran en la Ciudad de Buenos Aires y los suburbios adyacentes, en las principales ciudades del centro de la región pampeana y en los barrios cerrados de las capitales provinciales. Por otro lado, cerca de un tercio de la población del país constituye una clase media o media-baja estancada, un estrato que incluye a los pequeños comerciantes, trabajadores y empleados con nivel educativo medio o bajo, pensionistas y algunos profesionales independientes, cuyos ingresos se encuentran por encima de la línea de pobreza y que cuentan con cierta estabilidad laboral -incluyendo, en especial, la afiliación al sistema de seguridad social a través del trabajo-. Otro tercio de la población pertenece a la clase trabajadora -la exclase media empobrecida, trabajadores informales, trabajadores autónomos no calificados, trabajadores informales asalariados en micro-emprendimientos, trabajadores rurales o pequeños productores agropecuarios de regiones periféricas-, que es la principal usuaria de los servicios públicos de baja calidad y de la deteriorada infraestructura de educación y salud pública (Cassagne, 2004). Tienden a vivir 
en suburbios con mayores carencias de infraestructura del hogar y servicios o en grandes proyectos públicos de viviendas, particularmente en el noreste y noroeste del país. Finalmente, el último estrato se encuentra comprendido por los nuevos pobres y los excluidos. En este grupo, muchos hogares experimentan privaciones severas, déficits de infraestructura y servicios básicos, con mayores riesgos ambientales. Usualmente, su ingreso proviene de trabajos inestables o subsisten con la ayuda de programas de asistencia social. También hay una alta tasa de desempleo. Este segmento de la sociedad incluye a jóvenes que no han terminado el secundario, como también a niños que se encuentran en un estado de inseguridad alimentaria (Donza, 2019).

A pesar de la aproximación de la clasificación, debido a limitaciones en la información disponible, la distribución en la tabla podría ser compatible con la hipótesis de que existe un mayor grado de desigualdad social en la Argentina: las clases que se encuentran en los extremos de la estratificación social son más extensas que las otras. El índice Gini confirma que, efectivamente, la Argentina tal como todos los países sudamericanos- presenta un mayor nivel de desigualdad de distribución de ingresos con respecto a Italia, donde el índice Gini es bastante estable, alrededor de 35 puntos en la década del 2000; en la Argentina, en cambio, osciló desde 50 puntos en 2004 hasta 42 en años recientes (INDEC, 2018).

Para interpretar simultáneamente la relación entre ocupación y educación, se combinaron las dos dimensiones para crear una clasificación de catorce grupos (ver Tabla 3). Hubo algunos individuos clasificados como pertenecientes a las clases media y profesional sin título, respectivamente, que, considerando su nivel educativo y su número, fueron tratados como casos incongruentes y clasificados como pertenecientes a la clase trabajadora sin título. La construcción de grupos combinados permitió otorgar robustez estadística a los resultados modelo, debido a que los estimativos fueron basados en casos empíricamente observados y a que no se realizó ninguna inferencia sobre los casos no observados (Verbeek, 2008). 
Tabla 3

Tabla 3

Descripción de los grupos socio-económicos ("0" sin título, "l" nivel primario, "II" nivel secundario, "III" nivel terciario/universitario).

Porcentajes.

\begin{tabular}{|l|c|c|}
\hline & Argentina & Italia \\
\hline Marginal 0 & 4,4 & 0,1 \\
Marginal I & 8,1 & 3,6 \\
Marginal II & 3,0 & 2,8 \\
Marginal III & 0,8 & 0,5 \\
Clase trabajadora 0 & 2,5 & 0,4 \\
Clase trabajadora I & 18,4 & 21,8 \\
Clase trabajadora II & 13,0 & 16,6 \\
Clase trabajadora III & 4,2 & 1,8 \\
Clase media I & 5,3 & 8,8 \\
Clase media II & 12,7 & 26,3 \\
Clase media III & 13,8 & 11,2 \\
Clase media-alta y profesionales I & 0,2 & 0,4 \\
Clase media-alta y profesionales II & 0,9 & 2,4 \\
Clase media-alta y profesionales III & 12,7 & 3,1 \\
\hline Total & 100,0 & 100,0 \\
Casos válidos & 5823 & 5655 \\
\hline
\end{tabular}

La Tabla 4 evidencia la relación bivariada entre la salud percibida como "no buena" y los grupos socioeconómicos desfavorecidos. Particularmente, la salud es peor entre los grupos marginales y trabajadores. En ambos países, el porcentaje de los individuos en el grupo marginal sin título que declaran una salud desfavorable supera el 50\%. Estos grupos también presentan un mayor malestar psicológico (por debajo de la media).

Con el fin de probar la hipótesis sobre la relación entre la salud autopercibida y la condición socioeconómica fue necesario controlar por posibles factores de confusión. En este caso, se encontraban disponibles el sexo y la edad de los encuestados, factores de confusión fundamentales de la salud autopercibida. Así, se aplicó un modelo de regresión multivariada. Como la variable dependiente era dicotómica pero no muy asimétrica, se aplicó un modelo de regresión binaria (OLS) (Hellevik, 2007; King \& Zeng, 2001; Wooldridge, 2009). A su vez, se aplicó un modelo de regresión lineal en el segundo modelo para estimar las asociaciones entre las categorías socioeconómicas y el malestar psicológico. Las estimaciones asociadas con grupos sociales podrían ser directamente interpretadas como la probabilidad de declarar un peor estado de salud. A fin de dar robustez al análisis, se aplicó un modelo de regresión logística, el cual produjo resultados similares (ver Tabla A1 en el Apéndice). 
Tabla 4

Tabla 4

Grupos socioeconomicos según salud percibida como "no buena" y malestar psicologico (- peor, + mejor ; std). medias. Porcentajes y medias

\begin{tabular}{|l|c|c|c|c|}
\hline & $\begin{array}{c}\text { Argentina } \\
\%\end{array}$ & $\begin{array}{c}\text { Italia } \\
\%\end{array}$ & $\begin{array}{c}\text { Argentina } \\
\text { Media }\end{array}$ & $\begin{array}{c}\text { Italia } \\
\text { Media }\end{array}$ \\
\hline Marginal 0 & 57,9 & 50,0 & $-0,36$ & $-1,74$ \\
Marginal I & 47,1 & 38,9 & $-0,46$ & $-0,48$ \\
Marginal II & 50,0 & 39,4 & $-0,38$ & $-0,26$ \\
Marginal III & 36,7 & 40,0 & $-0,22$ & $-0,74$ \\
Clase trabajadora 0 & 52,8 & 43,5 & $-0,26$ & $-0,19$ \\
Clase trabajadora I & 42,2 & 34,1 & $-0,15$ & $-0,03$ \\
Clase trabajadora II & 34,9 & 27,2 & 0,05 & 0,00 \\
Clase trabajadora III & 34,4 & 25,3 & 0,06 & 0,05 \\
Clase media I & 42,1 & 31,2 & $-0,08$ & 0,05 \\
Clase media II & 33,9 & 26,1 & 0,14 & 0,04 \\
Clase media III & 28,1 & 25,1 & 0,24 & 0,09 \\
Clase media-alta y profesionales I & 27,3 & 28,0 & 0,10 & 0,02 \\
Clase media-alta y profesionales II & 23,6 & 18,2 & 0,34 & 0,13 \\
Clase media-alta y profesionales III & 29,6 & 18,4 & 0,33 & 0,20 \\
\hline Total & 37,6 & 28,9 & 0,00 & 0,00 \\
\hline
\end{tabular}

\section{Análisis}

Los resultados del análisis se encuentran plasmados en la Tabla 5. Tal como es de esperar, los grupos que ocupan las mejores posiciones socioeconómicas tienden a declarar un mejor estado de salud con respecto a los otros grupos, controlado por edad y sexo. Considerando la categoría de referencia de profesionales con educación terciaria/universitaria, la probabilidad de que los grupos marginales declaren peor salud es del $10-23 \%$ en la Argentina y del $12-33 \%$ en Italia. La probabilidad de que las clases trabajadoras declaren peor salud es aproximadamente del 5-16\% en la Argentina y no es significativa en Italia. Este resultado parecería ser bastante contradictorio, pero cabe señalar que las estimaciones específicas son positivas -las clases trabajadoras tienen una salud media peor- y que los intervalos de confianza son muy amplios, probablemente debido a la heterogeneidad de los grupos analizados.

La clase media tiene una probabilidad levemente positiva de manifestar peor salud autopercibida. La Figura 1 resume los resultados del análisis, mostrando intervalos creíbles que deben ser considerados como rangos dentro de los cuales hay una gran posibilidad ( $90 \%)$ de encontrar el parámetro real de la población. Los gradientes socioeconómicos de ambos países son fuertes y se asemejan entre sí. En particular, hay una diferencia evidente, especialmente en la Argentina, entre los grupos ocupacionales, la cual también se refleja al analizarla por los distintos niveles educativos dentro de dichos grupos. En detalle, los grupos con menor nivel educativo tienden a declarar tener un peor estado de salud que los demás. Asimismo, aquellos que han alcanzado un nivel educativo terciario/ universitario resultan más protegidos en todos los segmentos ocupacionales. 
Tabla 5

Tabla 5

Coeficientes de regresión binaria sobre la probabilidad de de declarar la propia salud como "No buena" vs. "Buena" y errores estándar

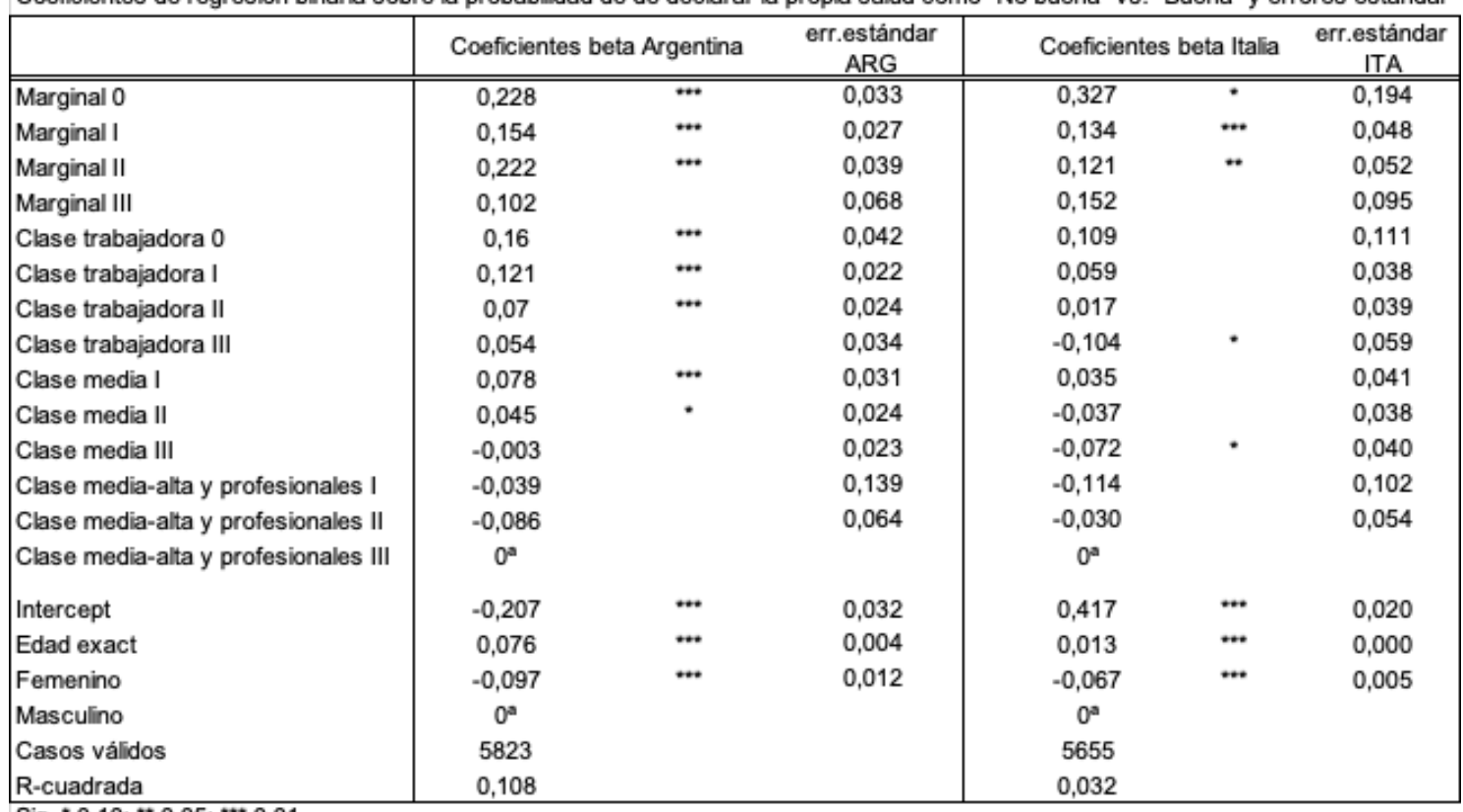

Sig. ${ }^{*} 0.10 ;{ }^{* *} 0.05 ;{ }^{\cdots *} 0.01$

a Categorias de referencia

Figura 1

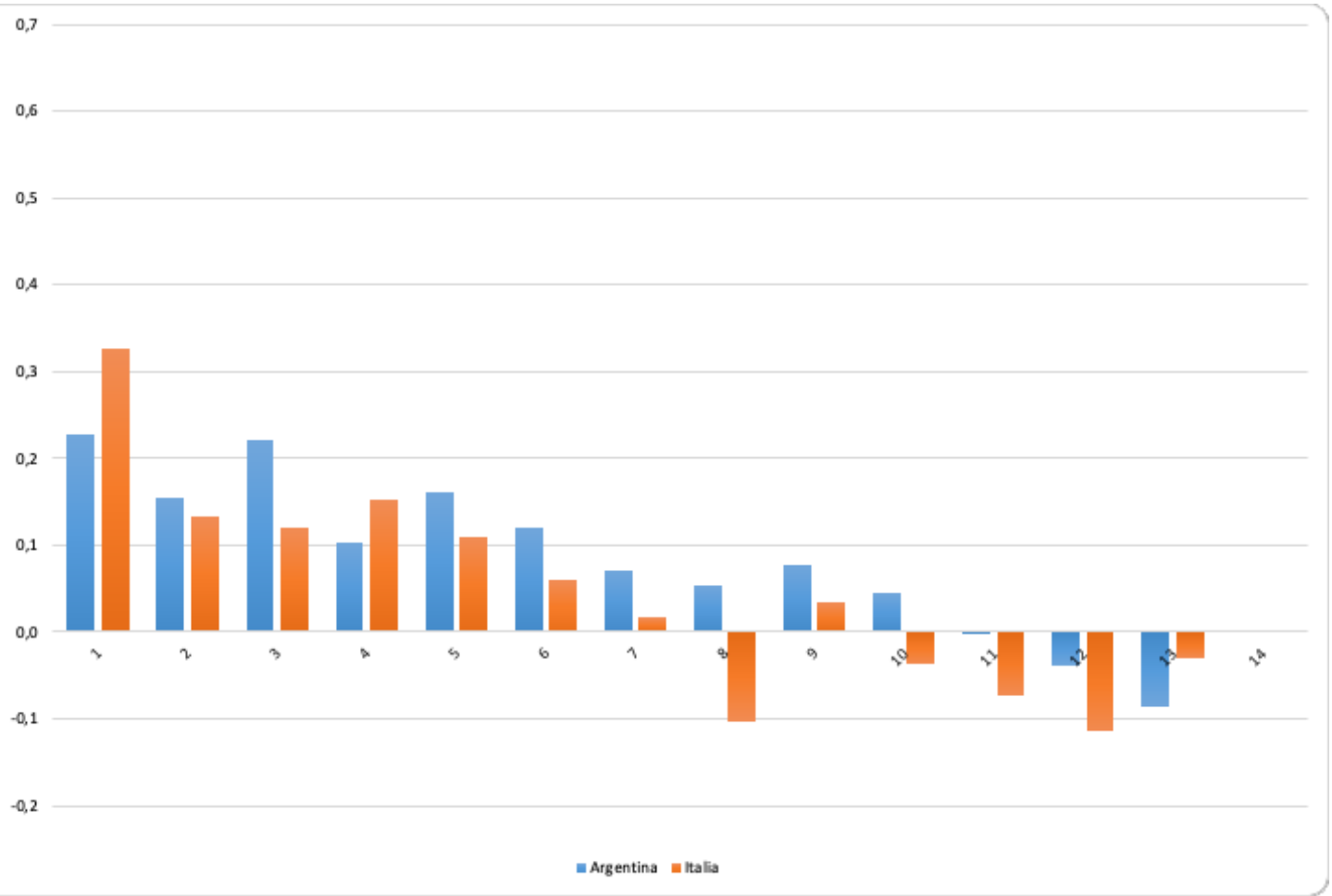

Probabilidad de declarar peor salud: intervalos 90 \% creíbles (bayesianos, bajo la presunción de distribución uniforme). Categoría de referencia: Clase media-alta y profesional III (nivel terciario completo)

La Tabla 6 y la Figura 2 muestran los resultados del análisis que concierne al índice de malestar psicológico. Como se sugirió, este indicador es utilizado para controlar el análisis previo. La correlación entre la salud autopercibida y el malestar psicológico es ya muy conocida y bastante obvia, ya que la primera 
es un indicador más general del bienestar. Los resultados aluden a un patrón similar, de acuerdo con la evidencia previa: las categorías sociales que presentan mayores desventajas son las que evidencian peores condiciones psicológicas, controlado por edad y sexo. Las clases marginal y trabajadora sin título exhiben coeficientes similares en ambos países. Sin embargo, algunas particularidades no son insignificantes. En general, las desigualdades en la Argentina parecen ser más pronunciadas que en Italia, y los diferenciales con la categoría de referencia son, en muchos casos, más intensos. Al considerar las categorías socioeconómicas marginales, parecería que en Italia la educación juega un rol más importante en las disparidades. Dentro de estas categorías, los italianos con nivel educativo terciario/universitario presentan un menor malestar psicológico. Pero, en general y observando las otras categorías, la asociación entre el estatus socioeconómico y el malestar psicológico parece estar más influido por la educación en la Argentina que en Italia; en Italia, los diferenciales para las clases trabajadora y media con respecto a la clase más alta son menos importantes. Estos resultados confirman un estudio precedente, llevado a cabo con datos de los años 2013-2016 (Sarti \& Rodríguez Espínola, 2018).

Tabla 6

Tabla 6

Coeficientes de regresión (OLS) sobre mejores condiciones psicológica y errores estándar

\begin{tabular}{|c|c|c|c|c|c|c|}
\hline & Coeficientes beta & vrgentina & $\begin{array}{l}\text { err.estánd } \\
\text { ar ARG }\end{array}$ & Coeficie & Italia & $\begin{array}{l}\text { err.estánd } \\
\text { ar ITA }\end{array}$ \\
\hline Marginal 0 & $-0,653$ & $\cdots$ & 0,070 & $-0,771$ & $*$ & 0,380 \\
\hline Marginal I & $-0,747$ & $\cdots$ & 0,057 & $-0,681$ & $\cdots$ & 0,102 \\
\hline Marginal II & $-0,659$ & $\cdots$ & 0,082 & $-0,497$ & $\cdots$ & 0,108 \\
\hline Marginal III & $-0,511$ & $\cdots$ & 0,144 & $-0,739$ & $\cdots$ & 0,207 \\
\hline Clase trabajadora 0 & $-0,562$ & $\cdots$ & 0,089 & $-0,383$ & * & 0,227 \\
\hline Clase trabajadora I & $-0,475$ & $\cdots$ & 0,046 & $-0,199$ & $*$ & 0,080 \\
\hline Clase trabajadora II & $-0,266$ & $\cdots$ & 0,050 & $-0,142$ & * & 0,081 \\
\hline Clase trabajadora III & $-0,228$ & $\cdots$ & 0,072 & $-0,117$ & & 0,126 \\
\hline Clase media I & $-0,401$ & $\cdots$ & 0,066 & $-0,185$ & $*$ & 0,087 \\
\hline Clase media II & $-0,189$ & * & 0,050 & $-0,106$ & & 0,079 \\
\hline Clase media III & $-0,075$ & & 0,049 & $-0,062$ & & 0,084 \\
\hline Clase media-alta y profesionales I & $-0,206$ & & 0,292 & $-0,046$ & & 0,211 \\
\hline Clase media-alta y profesionales II & 0,015 & & 0,135 & $-0,035$ & & 0,113 \\
\hline Clase media-alta y profesionales III & $0^{*}$ & & & $0^{*}$ & & \\
\hline Intercept & 0,377 & $\cdots$ & 0,068 & 0,465 & $\cdots$ & 0,098 \\
\hline Edad exact & $-0,024$ & $\cdots$ & 0,008 & $-0,052$ & $\cdots$ & 0,008 \\
\hline Femenino & 0,240 & $\cdots$ & 0,026 & 0,187 & $\cdots$ & 0,027 \\
\hline Masculino & $0^{*}$ & & & $0^{*}$ & & \\
\hline Casos válidos & 5823 & & & 5655 & & \\
\hline R-cuadrada & 0,075 & & & 0,034 & & \\
\hline
\end{tabular}


Figura 2

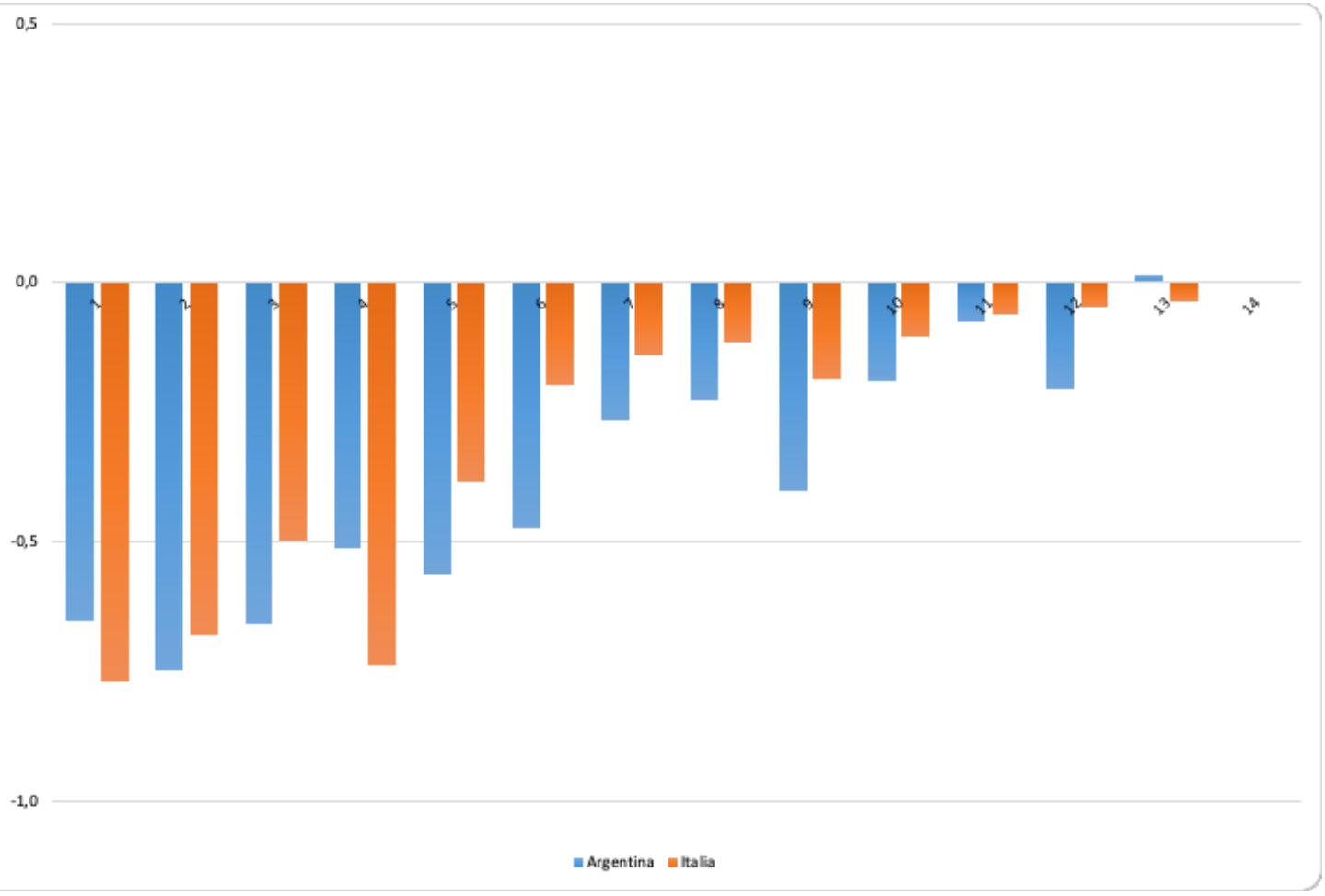

Probabilidad de mejor condición de salud: intervalos $90 \%$ creíbles (bayesianos, bajo la presunción de distribución uniforme). Categoría de referencia: Clase media-alta y profesional III (nivel terciario completo)

\section{Conclusiones}

En el presente estudio se han comparado las desigualdades en salud entre Italia y la Argentina. La evidencia en Sudamérica referente a este tema es escasa, mientras que la situación de los países de la OCDE es mejor conocida e investigada más extensamente (Marmot, 2013; OMS, 2013). Por ejemplo, las desigualdades en salud de Italia son comparables a las de los otros países mediterráneos, ligeramente menores que las de los países escandinavos y significativamente inferiores que las de los países de Europa Oriental. Sin embargo, como se destacó anteriormente, las desigualdades en salud también son sustanciales en Italia.

Con respecto a esto, una importante pregunta de investigación es si el patrón de desigualdades en salud es constante entre contextos y ambientes sociales diferentes (Marmot, 2017). Esta es la cuestión que se encuentra en la base de la comparación de los dos países considerados aquí.

La principal dificultad que se presenta al responder a esta pregunta es la ausencia de conjuntos de datos comparables. Por ejemplo, la Argentina no se encuentra incluida en la Encuesta Mundial de Salud de la OMS.

A tal efecto, se creó un conjunto de datos comparables utilizando dos importantes encuestas nacionales: la EDSA, de los años 2017 y 2018, y "EHISISTAT del año 2015”. La armonización de los indicadores de ambas encuestas no fue sencilla porque fueron concebidas en distintos términos y con objetivos disímiles. La encuesta argentina se centra en la pobreza y las condiciones sociales, mientras que la italiana trata el tema de las condiciones de salud y la utilización del sistema sanitario. 
Sin embargo, a pesar de las limitaciones en la armonización de los indicadores, se han creado algunas variables que sirvieron para comparar la desigualdad en salud en los dos países: la salud autopercibida, un índice de malestar psicológico, el nivel educativo y la condición ocupacional. En la primera parte de este trabajo, el resumen descriptivo del contexto de salud mostró que la Argentina e Italia poseen distintos niveles de bienestar, mortalidad y desempeño de los servicios de salud.

No obstante, en la segunda parte, los análisis específicos de la relación entre la salud individual y las condiciones socioeconómicas sugirieron un patrón de desigualdades relativas entre ambos países: en la Argentina se observó mayor capacidad explicativa de los factores socioeconómicos, laborales y educativos. Sin duda, la marginalidad socioeconómica y el bajo nivel educativo juegan una parte extremadamente importante en la generación de desigualdades. En efecto, considerando al índice de malestar psicológico, se observó que en Italia el nivel educativo parece tener mayor peso en las clases media, trabajadora y en las categorías socioeconómicas marginales. En cambio, el análisis plantea que en la Argentina el rol de la educación explica mejor las diferencias en la salud. En este sentido, a pesar de las limitaciones de los datos y el análisis, los descubrimientos parecen confirmar la hipótesis de Marmot (2007) sobre la configuración general de las desigualdades en salud, también para la Argentina. En particular, las clases marginales y trabajadoras - trabajadores manuales, empleados precarizados, desempleados, individuos sin educación- sistemáticamente presentan peores condiciones de salud con respecto a las clases medias, mientras que los miembros de la clase alta y los profesionales ocupan la mejor posición del gradiente.

En síntesis, los resultados obtenidos son coherentes con las teorías estructuralistas explicadas en la primera parte del artículo. El estatus socioeconómico individual basado en la posición ocupacional y el nivel educativo alcanzado parece estrechamente asociado a los procesos de deterioro de la salud: estrés constante, privación material, desventajas acumuladas en el curso de la vida y déficit de capital cultural. En otras palabras, los individuos que se encuentran en áreas de marginalidad o vulnerabilidad social son más propensos a tener un peor estado de salud. En esta perspectiva, la atenuación de las desigualdades en salud puede lograrse por medio de la reducción de las disparidades ocasionadas por la estratificación social general.

\section{Aprobación ética}

Los datos de Italia fueron recolectados por ISTAT- Instituto Nacional de Estadística (Italia), de acuerdo con los estándares internacionales y con la legislación italiana (Art. 9 del dlgs. N. 322/89; dlgs. N. 196/03). Más información en: http://www.istat.it/en/privacy.

Los datos de la Argentina fueron recolectados por el Observatorio de la Deuda Social Argentina-UCA (Pontificia Universidad Católica Argentina). Todos los procedimientos empleados respetaron los lineamientos éticos recomendados para la investigación con humanos por la declaración de Helsinki y la Ley Nacional 25326 de Protección de los Datos Personales de acuerdo con la legislación argentina. Más información, en http://www.uca.edu.ar/index.php/site/index/es/uca/observatorio-de-la- 
deuda-social-argentina/encuesta-de-la-deuda-social/anexo-metodologicoencuesta-deuda-social/.

\section{Reconocimientos}

El presente artículo fue elaborado en el contexto de la Red INCASI, coordinada por el Dr. Pedro López-Roldán, un proyecto europeo que ha recibido financiación del programa de investigación e innovación Horizonte 2020 de la Unión Europea, bajo el Maria Sklodowska-Curie GA N 691004. Este artículo refleja únicamente la opinión de los autores, y la agencia no es responsable de ningún uso que se haga de la información que contiene.

This article was elaborated in the context of INCASI Network, a European project that has received funding from the European Union's Horizon 2020 research and innovation programme under the Marie Sklodowska- Curie GA No 691004 and coordinated by Dr. Pedro López- Roldán. This article reflects only the author's view and the Agency is not responsible for any use that may be made of the information it contains.

\section{Referencias}

Aittomäki, A., Martikainen, P., Laaksonen, M., Lahelma, E. \& Rahkonen, O. (2012). Household economic resources, labour-market advantage and health problemsA study on causal relationships using prospective register data. Social Science \& Medicine, 75(7), 1303-1310.

Aneshensel, C. S. (1992). Social stress: Theory and research. Annual Review of Sociology, $18,15-38$.

Banco Mundial (2016). World Development Indicators 2016. Recuperado de: https://openknowledge.worldbank.org/bitstream/handle/10986/23969/97 81464806834.pdf

Barber, R. M., Fullman, N., Sorensen, R. J., Bollyky, T., McKee, M., Nolte, E. et al. (2017). Healthcare access and quality index based on mortality from causes amenable to personal health care in 195 countries and territories, 1990-2015: A novel analysis from the Global Burden of Disease Study 2015. The Lancet, 390 (10091), 231-266.

Bartley, M. (2003). Commentary: Relating social structure and health. International Journal of Epidemiology, 32(6), 958-960.

Bartley, M. (2004). Health inequality: An introduction to concepts, theories and methods. Cambridge: Polity Press.

Beckett, M. (2000). Converging health inequalities in later life: An artifact of mortality selection? Journal of Health and Social Behavior, 41(1), 106-119.

Brunner, E. \& Marmot, M. (2006). Social organization, stress, and health. En M. Marmot \& R. G. Wilkinson (Eds.). A cura disocial determinants of health (6-30). Nueva York: Oxford University Press.

Cassagne, J. C. (2004). El futuro de los servicios públicos. Revista de Derecho Administrativo Económico, 12(1), 1-16.

Cassel, J. (1976). The contribution of the social environment to host resistance. American Journal of Epidemiology, 104(2), 107-123. 
Cohen, S., Janicki-Deverts, D. \& Miller, G. E. (2007). Psychological stress and disease. JAMA, 298(14), 1685-1687.

Collins, C., McCartney, G., \& Garnham, L. (2015). Neoliberalism and health inequalities. In K. E. Smith, C. Bambara, \& S. E. Hill (Eds.), Health inequalities: Critical perspectives (pp.124-137). Oxford: Oxford University Press

Cullati, S., Rousseaux, E., Gabadinho, A., Courvoisier, D. S. \& Burton-Jeangros, C. (2014). Factors of change and cumulative factors in self-rated health trajectories: A systematic review. Advances in Life Course Research, 19, 14-27.

Della Bella, S., Sarti, S., Lucchini, M. \& Bordogna, M. T. (2011). A comparative analysis of inequality in health across Europe. Sociological Research Online, 16(4), 1-15.

Dalle, P. (2017). Climbing up a steeper staircase: Intergenerational class mobility across birth cohorts in Argentina (2003-2010). Research in Social Stratification and Mobility. http://dx.doi.org/10.1016/j.rssm.2017.12.002

De Irala-Estévez, J., Groth, M., Johansson, L., Oltersdorf, U., Prättälä, R. \& MartínezGonzález, M. A. (2000). A systematic review of socio-economic differences in food habits in Europe: Consumption of fruit and vegetables. European Journal of Clinical Nutrition, 54(9), 706-714.

DiPrete, T. A. \& Eirich, G. M. (2006). Cumulative advantage as a mechanism for inequality: A review of theoretical and empirical developments. Annual Review Of Sociology, 32, 271-297.

Donza, E. (2019). Heterogeneidad y fragmentación del mercado de trabajo (2010-2018). Ciudad Autónoma de Buenos Aires: Educa.

Drever, F., Daran, T. \& Whitehead, M. (2004). Exploring the relation between class, gender, and self- rated general health using the new socioeconomic classification. A study using data from 2001 census. Journal of Epidemiology and Community Health, 58(7), 590-596.

Dupre, M. E. (2008). Educational differences in health risks and illness over the life course: A test of cumulative disadvantage theory. Social Science Research, 37(4), 1253-1266.

Eikemo, T. E., Huisman, M., Bambra, C. \& Kunst, A. E. (2008). Health inequalities according to educational level in different welfare regimes: A comparison of 23 European countries. Sociology of Health \& Illness, 30(4), 565-582. http://dx.doi .org/10.1111/j.1467-9566.2007.01073.x

Erikson, R. \& Goldthorpe, J. H. (1992). The Constant flux. Oxford: Clarendon Press.

Goldthorpe, J. H. (2010). Analysing social inequality: A critique of two recent contributions from economics and epidemiology. European Sociological Review, 26(6), 731-744.

Hellevik, O. (2007). Linear versus logistic regression when the dependent variable is a dichotomy. Quality \& Quantity, 43(1), 59-74.

Herzlich, C. \& Adam, P. (1994). Sociologie de la maladie et de la medicine. París: Nathan.

Idler, E. L. \& Benyamini, Y. (1997). Self-rated health and mortality: A review of twenty seven community studies. Journal of Health and Social Behavior, 38(1), 21-37.

ISTAT (2019). Il Benessere Equo e Sostenible in Italia. Recuperado de: https://www.is tat.it/it/files//2019/12/Bes_2019.pdf

Johnson, R. J. \& Wolinsky, F. D. (1993). The structure of health status among older adults: Disease, disability, functional limitation, and perceived health. Journal of Health and Social Behavior, 34(2), $105 \mathrm{e}$.

Jylhä, M. (2009). What is self-rated health and why does it predict mortality? Towards a unified conceptual model. Social Science \& Medicine, 69(3), 307-316. 
Karasek, R. (1979). Job demands, job decision latitude, and mental strain: Implications for job redesign. Administrative Science Quarterly, 24(2), 285-308.

Karjalainen, A. \& Niederlaender, E. (2004). Statistics in focus. Population and social conditions. Luxemburgo: EUROSTAT.

King, G. \& Zeng, L. (2001). Logistic regression in rare events data. Political Analysis, 9(2), 137-163.

Kitigawa, E. M. \& Hauser, P. M. (1973). Differential's mortality in the United States: A study of socioeconomic epidemiology. Cambridge, MSS: Harvard University Press.

Kuh, D. \& Ben-shomo, Y. (1997). A life course approach to chronic disease epidemiology. Oxford: Oxford University Press.

Mancino, L., Lin, B. H. \& Ballenger N. (2004). The role of economics in eating choices and weight outcomes. Agriculture information bulletin, $\mathrm{N}^{\circ}$ n. 791 .

Marmot, M. (2013). Health inequalities in the EU - Final report of a consortium. http: //dx.doi.org/10.2772/34426.

Marmot, M. (2017). The health gap: The challenge of an unequal world: The argument. International Journal of Epidemiology, http://dx.doi.org/10.1093/ije/dyx163

McLaren, L. (2007). Socioeconomic status and obesity. Epidemiologic Reviews, 29, 2948. http://dx.doi.org/10.1093/epirev/mxm001

Mulatu, M. S. \& Schooler, C. (2002). Casual connections between socio-economic status and health: Reciprocal effects and mediating mechanism. Journal of Health and Social Behavior, 43(1), 22-41.

Navarro, V. (1986). Crisis, health, and medicine: A social critique. Nueva York: Tavistock.

Phelan, J., Link, B. G. \& Tehranifar, P. (2010). Social conditions as fundamental causes of health inequalities: Theory, evidence, and policy implications. Journal of Health and Social Behavior, 51(1), S28-S40.

OMS (2013). Review of social determinants and the health divide in the WHO European region: Final report. Copenhague: WHO Regional Office for Europe.

OMS (2015). State of inequality: Reproductive, maternal, newborn and child health. Ginebra: World Health Organization.

OMS (2017). WHO Report on the Global Tobacco Epidemic, 2017: Monitoring tobacco use and prevention policies. Ginebra: World Health Organization.

Rodrik, D. (2016). Premature deindustrialization. Journal of Economic Growth, 21(1). $1-33$.

Rodríguez Espínola, S. (2017). La salud en población urbana argentina desde una mirada multidimensional de la pobreza [en línea]. Universidad Católica Argentina. Observatorio de la Deuda Social Argentina. Barómetro de la Deuda Social Argentina. Serie del Bicentenario (2010-2016), año 7. Disponible en: http://bibliotecadigital.uca.edu.ar/repositorio/investigacion/salud-poblacio n-urbana-argentina-espinola.pdf [Fecha de consulta: 04/05/2020].

Ross, C. E. \& Mirowsky, J. (1999). Refining the association between education and health: The effects of quantity, credential, and selectivity. Demography, 36(4), 445-460.

Ross, C. E. \& Wu, C. (1996). Education, age, and the cumulative advantage in health. Journal of Health and Social Behavior, 37(1), 104-120.

Sandleris, G. \& Wright, M. L. (2014). The costs of financial crises: Resource misallocation, productivity, and welfare in the 2001 argentine crisis. The Scandinavian Journal of Economics, 116(1), 87-127. 
Sarti, S. \& Zella, S. (2016). Changes in the labour market and health inequalities during the years of the recent economic downturn in Italy. Social Science Research, 57, 116-132.

Sarti, S. \& Rodríguez Espínola, S. (2018). Health inequalities in Argentina and Italy: A comparative analysis of the relation between socio-economic and perceived health conditions. Research in Social Stratification and Mobility, 55, 89-98.

Schiavone, M. A., Rodríguez Espínola, S., Seoane, H. y Ríos, J. (2015). Salud percibida. Cobertura, utilización y acceso al sistema de atención de la salud en el área metropolitana de Buenos Aires. Buenos Aires: EDUCA.

Siegrist, J. (1999). A theory of occupational stress. En J. Dunham (Ed.). Stress in occupations: Past, present, and future (pp. 63-66). Londres: Whurr Publisher Limited.

Siegrist, J. (2000). Place, social exchange and health: Proposed sociological framework. Social Science and Medicine, 52(9), 1283-1293.

Siegrist, J. \& Marmot, M. (2004). Health inequalities and the psychosocial environment - two scientific challenges. Social Science \& Medicine, 58(8), 1463-1473.

Spencer, N. \& Logan, S. (2002). Social influences on birth weight. Archives of Disease in Childhood - Fetal and Neonatal Edition, 86, F6-F7. http://dx.doi.org/10.113 6/fn.86.1.F6

Tucker-Drob, E. M., Rhemtulla, M., Harden, K. P., Turkheimer, E. \& Fask, D. (2011). Emergence of a Gene x Socioeconomic Status Interaction on Infant Mental Ability Between 10 Months and 2 Years. Psychological Science, 22(1), 125-133. h ttps://doi.org/10.1177/0956797610392926

Turkheimer, E., Haley, A., Waldron, M., D’Onofrio, B. \& Gottesman, I. I. (2003). Socioeconomic Status modifies heritability of IQ in Young Children. Psychological Science, 14(6), 623-628.

Verbeek, M. (2008). A guide to modern econometrics. Chichester: John Wiley \& Sons.

Wilkinson, R. (2002). Commentary: Liberty, fraternity, equality. International Journal of Epidemiology, 31(3), 538-543.

Wilkinson, R. \& Marmot, M. (2003). Social determinants of health: The solid facts. Copenhague,: World Health Organization.

Willson, A. E., Shuey, K. M. \& Elder, G. H. (2007). Cumulative advantage processes as mechanism of inequality in life course health. American Journal Of Sociology, 112(6), 1886-1924.

Wooldridge, J. (2009). Introductory econometrics: A modern approach. Mason: SouthWestern. 


\section{Apéndice A}

Tabla A1

Coeficientes de regresión logistica (logit) de la propensión a declarar salud "No buena" vs "Buena" y errores estándar

\begin{tabular}{|c|c|c|c|c|}
\hline & Coeficientes beta Argentin & Coeficientes beta Italia & err.estándar ARG & err.estándar ITA \\
\hline Marginal 0 & 1,033 & 19,622 & 0,157 & 0,000 \\
\hline Marginal I & 0,719 & 0,678 & 0,129 & 0,234 \\
\hline Marginal II & 1,027 & 0,601 & 0,180 & 0,249 \\
\hline Marginal III & 0,505 & 0,760 & 0,319 & 0,490 \\
\hline Clase trabajadora 0 & 0,728 & 0,528 & 0,194 & 0,543 \\
\hline Clase trabajadora I & 0,575 & 0,280 & 0,106 & 0,172 \\
\hline Clase trabajadora II & 0,347 & 0,079 & 0,116 & 0,174 \\
\hline Clase trabajadora III & 0,278 & $-0,441$ & 0,163 & 0,260 \\
\hline Clase media I & 0,375 & 0,163 & 0,147 & 0,188 \\
\hline Clase media II & 0,218 & $-0,160$ & 0,117 & 0,168 \\
\hline Clase media III & $-0,021$ & $-0,307$ & 0,117 & 0,179 \\
\hline Clase media-alta y profesionales I & $-0,263$ & $-0,485$ & 0,720 & 0,443 \\
\hline Clase media-alta y profesionales II & $-0,446$ & $-0,135$ & 0,338 & 0,241 \\
\hline Clase media-alta y profesionales III & $0^{\mathrm{s}}$ & $0^{\mathrm{a}}$ & & \\
\hline Intercept & $-3,709$ & $-0,650$ & 0,162 & 0,211 \\
\hline Edad exact & 0,347 & 0,154 & 0,018 & 0,018 \\
\hline Femenino & 0,462 & 0,218 & 0,058 & 0,058 \\
\hline Masculino & $0^{\infty}$ & $0^{a}$ & & \\
\hline Casos válidos & 5823 & 5655 & & \\
\hline Pseudo R-cuadrado (McFadden) & 0,085 & 0,025 & & \\
\hline
\end{tabular}

